\title{
Design of a Greenhouse Video Monitoring Platform Based on Cloud Computing
}

\author{
Cuihua Sun ${ }^{\mathrm{a}}$, Feng Wang ${ }^{b^{*}}$, Jun Zou ${ }^{\mathrm{c}}$ and Qianghe Qun ${ }^{\mathrm{d}}$ \\ Smart Agriculture collage, Suzhou Polytechnic Institute of Agriculture, Suzhou 215008, China

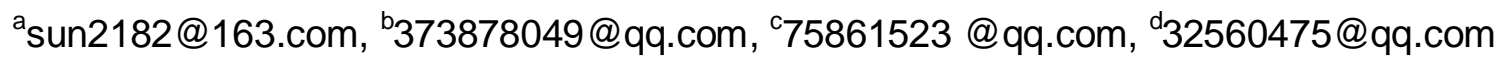 \\ * The Corresponding Author
}

Keywords: Cloud computing; Video surveillance; Greenhouse monitoring; Intelligent agriculture

\begin{abstract}
With the advancing of agricultural modernization, agricultural science and Technology Park, modern agricultural park for greenhouse monitoring system use continues to expand the scale of the growing number of monitoring equipment, agricultural expert system requires more HD video camera, retention time history data inquiry and comparison for longer, which put forward higher requirements for video storage. This paper proposes clouds-based greenhouse video monitoring platform and builds the platform's architecture and data flow. The experiment proves that it can effectively improve the processing efficiency of video.
\end{abstract}

\section{Introduction}

With the vigorous development of China's agricultural informatization, greenhouse remote monitoring system has been greatly improved in technology and scale but compared with the remote monitoring system of some developed countries, there is a certain gap between the degree of perfection of Intelligent Greenhouse in our country, the stability of production facilities and the level of modernization. The 2016 central document, "vigorously promote the" Internet plus "modern agriculture, application networking, cloud computing, big data, mobile Internet and other modern information technology, promote the upgrading of agricultural industry chain [1]. " "Internet plus" represents the development of modern agriculture in a new direction and new trends, but also provides a new method for the path and the transformation of agricultural development mode. With the continuous development of network technology and intelligent mobile phone, provides a good hardware platform and high-speed communication network foundation for the development of Intelligent Greenhouse Monitoring System in our country[2], the use of intelligent mobile phone remote monitoring has become a major trend of intelligent greenhouse monitoring, the greenhouse workers not at the scene of the case, also can receive from the greenhouse environmental information and alarm status and control the growth whenever and wherever possible, the camera to see the crops[3].

\section{Video Storage Technology}

The Current Situation of video Storage Technology. With the development of technology, distributed cloud storage [4] becomes more and more important, and some medium scale video monitoring systems have adopted distributed cloud storage scheme. According to the investigation in many modern agricultural parks, most of the greenhouse video monitoring systems adopt distributed storage mode, and monitoring centers seldom install specialized storage servers. The new wireless video monitoring system based on $4 \mathrm{G}$ router has a special storage server in the local area, and a storage server is installed in the monitoring center. The current storage is basically the way of direct storage, which is to connect several disks on the server, using point to point disk system, the storage scalability and storage performance is difficult to improve. With the construction of high-definition video surveillance system[5], many new functions of digital monitoring generated by digital monitoring instead of traditional technology are becoming mainstream technology. The DVR, the core device of the original digital monitoring, is gradually 
reduced and is replaced by a video server with IP SAN as the core of the monitoring system[6].

The Application of Video Surveillance System in Modern Agriculture. China is a large agricultural country, and the development of information - based greenhouse has unparalleled resource advantages and potential. However, greenhouse greenhouses in China are still at the initial stage. There are many problems such as unreasonable structure, serious waste of resources, high cost of operation and management, low level of management and low output per unit area. Therefore, an unattended monitoring and management system is urgently needed to monitor and manage the greenhouse in real time and dynamically. In recent years, with the popularization and development of communication technology, the rise of the Internet technology and embedded technology, the embedded monitoring system based on wireless sensor network technology and has a more extensive application of space, especially in recent years the popularity of the 4G network, provides the conditions for the formation of large-scale remote monitoring, remote users can through the network the greenhouse for real-time monitoring and management, and video monitoring system in network compression technology and network transmission technology, realize the unmanned on duty in the greenhouse.

This is an intelligent greenhouse video monitoring system in the agricultural park, which can use the mobile phone to see the growth of plants in real time as shown in the Fig. 1.

\section{The Key Technology}

This design takes cloud computing framework as development platform, and uses wireless communication network's mobile terminal device to transmit data, and realizes the design of remote monitoring for mobile terminal devices. This design can monitor the environment of greenhouse, improve work efficiency, and increase user's flexibility to use terminal equipment.

Platform Overall Structure. The hardware part of the design of the video collection module based on cloud computing is composed of two parts: the main control chip and the external expansion[8]. The expansion includes the LCD display section, the USB controller, the camera, the network transmission, etc. The related software part of the design includes Linux system, USB camera driver, video acquisition program, real-time display program, network transmission program and so on. The overall structure is as shown in the Fig. 2.

Construction of Cloud Computing Cluster. By utilizing the advantage of cloud computing cluster, we first build cluster, distribute a task thread by a Master node, and are executed by several Slave.

Web Server Side Display. Video monitoring system for greenhouse based on cloud computing in front of the collected video information through the cloud computing architecture stored in a distributed file system, check the data information, the parallel computing is distributed to multiple servers, so the computational efficiency is improved obviously. 


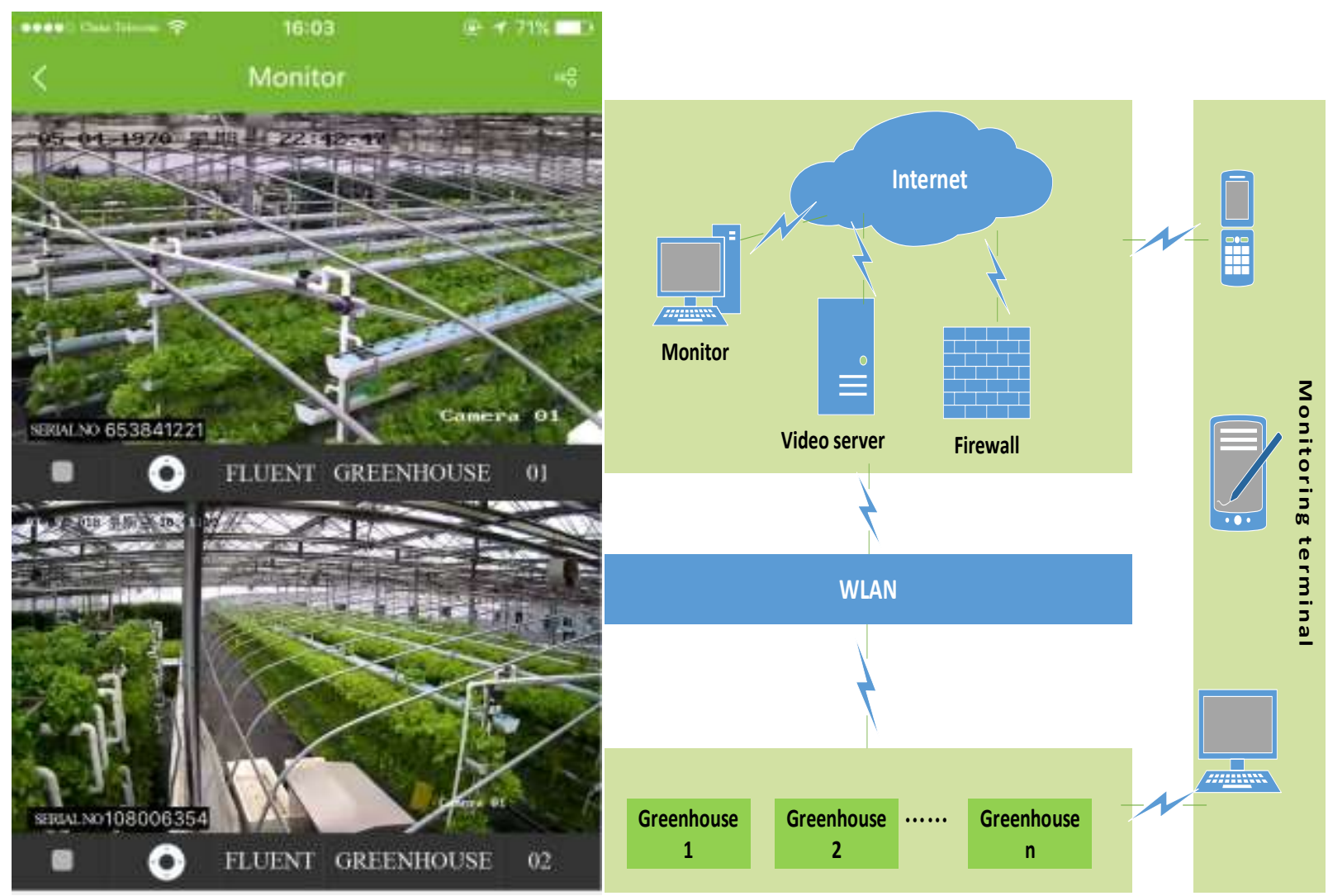

Figure 1. Intelligent greenhouse video monitoring system

Figure 2. The overall structure

\section{Simulation Experiment}

In order to prove the video storage scheme based on cloud computing flow performance advantages of high concurrent write in multi-channel video, 30 -300 Road, a resolution of $1080 \mathrm{P}$ video streams are concurrent write based on cloud storage server and storage server based on the file system, each video flow rate constant is $4 \mathrm{Mbit} / \mathrm{s}$, average write speed is measured as shown in the Fig. 3 .

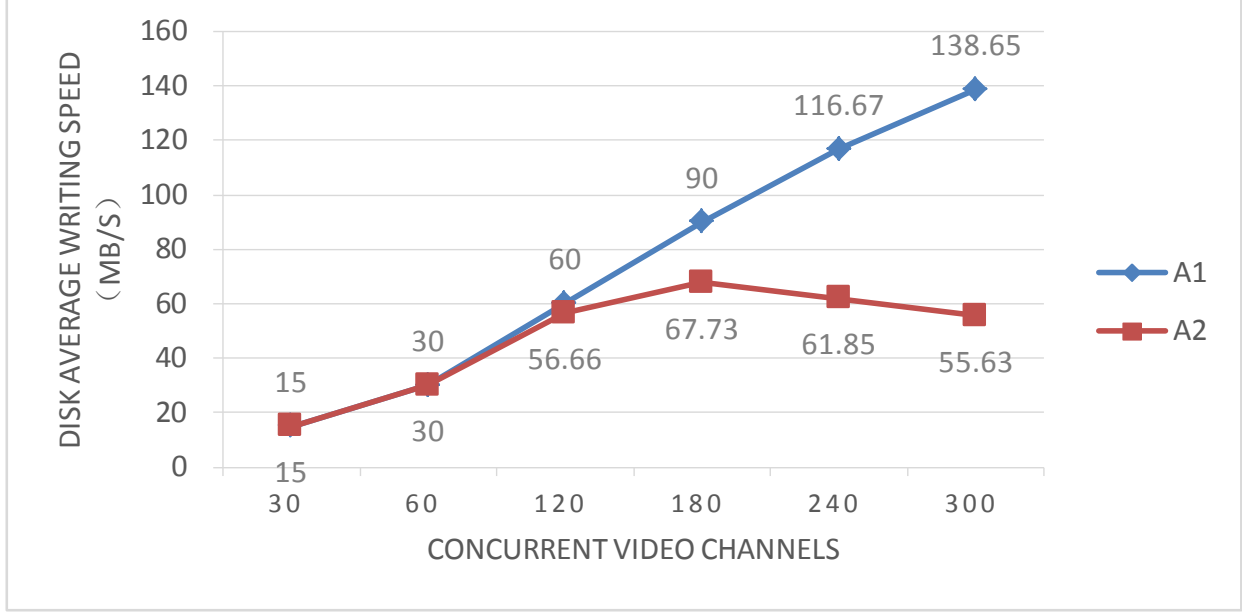

Figure 3. Performance comparison evaluation

When the video number was 30 and 60 , the total input bandwidth were $15 \mathrm{MB} / \mathrm{s}$ and $30 \mathrm{MB} / \mathrm{s}$, based on the video storage scheme of cloud computing and storage scheme based on file system write speed and input the total bandwidth. But when the video number is 120 , the input bandwidth of $60 \mathrm{MB} / \mathrm{s}$, video storage scheme based on cloud computing successfully real time to write, and write speed storage file system program only based on $56.66 \mathrm{MB} / \mathrm{s}$, is lower than the input bandwidth, resulting in a delayed write. Some video number rises to 300 , the total input bandwidth of $150 \mathrm{MB} / \mathrm{s}$, the cloud storage server is able to real-time video data will be written to disk based 
storage scheme and write speed of file system based on slightly down to $55.63 \mathrm{MB} / \mathrm{s}$. with video channels continue to rise, based on the write speed video storage scheme for cloud computing steady As a result, the writing speed of file system based storage scheme has decreased significantly. So we can see that the video storage scheme based on cloud computing is more stable than file system based storage scheme.

\section{Conclusions}

The massive video conversion platform based on cloud computing, which provides a new idea for solving the video conversion problems, by combining a variety of open source cloud platform, and the two was developed on the basis of the platform, write the core service routines, and optimize the scheduling strategy of platform, effectively improves the processing efficiency of the video business transformation.

\section{Acknowledgements}

This work was sponsored by Qing Lan Project, supported by the education "13th Five-Year" Planning issues of Jiangsu province (No. B-b/2016/03/41), the research subject of higher education reform in Jiangsu (No. 2017JSJG348), the modern educational technology research project of Jiangsu province (No. 2017-R-58821), and depth integration training platform of production and education of Jiangsu Province on 2016 (The depth integration training platform of intelligent digital plant factory production of production and education).

\section{References}

[1] Information on https://baike.baidu.com/item/\%E4\%B8\% A5?fr=aladdinE4\%BB\%B6.

[2] N. Valentini, A. Saponieri, L. Damiani: Ocean and Coastal Management, Vol. 32 (2017) No.3, p.122.

[3] Jiang M, Niu ZY, Zhang SP. Design and implementation of video surveillance storage system. Computer Engineering and Design, 2014,35(12):4195-4201 (in Chinese with English abstract). [doi: 10.3969/j.issn.1000-7024.2014.12.027]

[4] Wang YJ, Sun WD, Zhou S, Pei XQ, Li XY. Key technologies of distributed storage for cloud computing. Ruan Jian Xue Bao/ Journal of Software, 2012,23(4):962-986 (in Chinese with English

[5] K. Zhang, Z. Huang, S. L. Zhang: Ecological Informatics, Vol. 4 (2017) No.8, p.139.

[6] Sun ZZ, Zhang QX, Tan YA, Li YZ. Ripple-RAID: A high-performance and energy-efficient RAID for continuous data storage. Ruan Jian Xue Bao/Journal of Software, 2015,26(7):1824-1839 (in Chinese with English abstract). http://www.jos.org.cn/10009825/4606.htm [doi: 10.13328/j.cnki.jos.004606]

[7] W.H. Liu, Z Wang: Computer Technology and Its Applications, Vol. 41 (2015) No.6, p.139 (In Chinese).

[8] Y. Liu, G. Zhang, L. Hang: Computer Technology and Its Applications, Vol. 30 (2013) No.11, p.3331 (In Chinese). 